\title{
Yield of Tomato in Soil Treated with Compost, Amorphous Primary Minerals and Microorganisms
}

\author{
Juan Martín Parra Delgado, Edgar Quero Gutiérrez², Leopoldo Partida Ruvalcaba ${ }^{3 *}$, \\ Teresa de Jesús Velázquez Alcaraz', Tomás Díaz Valdés ${ }^{1}$, Felipe Ayala Tafoya ${ }^{1}$, \\ Marino Valenzuela López ${ }^{1}$ \\ ${ }^{1}$ Facultad de Agronomía, Universidad Autonoma de Sinaloa, Culiacán, México \\ ${ }^{2}$ Laboratorio de Análisis y Diagnóstico del Patrimonio, Colegio de Michoacán, La Piedad, México \\ ${ }^{3}$ Universidad Tecnológica de Culiacán, Culiacán de Rosales, México \\ Email: "parpolo@yahoo.com.mx
}

Received 25 August 2015; accepted 11 September 2015; published 17 September 2015

Copyright (C) 2015 by authors and OALib.

This work is licensed under the Creative Commons Attribution International License (CC BY). http://creativecommons.org/licenses/by/4.0/

(C) (i) Open Access

\section{Abstract}

This research was conducted to determine the chemical condition initial and final of the soil, having been treated with compost, primary minerals amorphous (MPA) and microorganisms (M) as well as response of the hybrid "Moctezuma" of saladette tomato (Lycopersicon esculentum Mill.). Compost and MPA were applied 30 days before the transplant, the Steiner solution from the transplant and the microorganisms every eight days up to harvest in drip irrigation; the population density was $\mathbf{2 5 , 0 0 0}$ plants per hectare, the which they were handled to a stem in separate rows 1.6 meters between they. The experimental design was randomized complete block with four

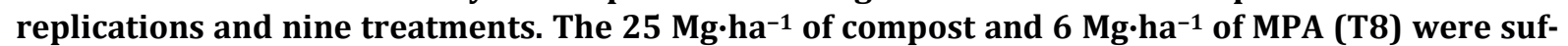
ficient to yield $114.0 \mathrm{Mg} \cdot \mathrm{ha}^{-1}$ with an increase of $9.3 \%$ compared to $104.3 \mathrm{Mg} \cdot \mathrm{ha}^{-1}$ achieved with Steiner solution. Compost, MPA and M were made that the soil increased the fertility from before transplantation, and until the harvest is continued providing enough nutrients, with the exception to $\mathrm{Cl}$ and $\mathrm{S}$, which were not detected after harvest. Deficiencies of $\mathrm{Cl}$ and $\mathrm{S}$ perhaps were one of the causes of that plants did not produced beyond that achieved with Steiner solution or $\mathbf{T}_{8}$, this latter that in turn allowed manage more sustainably the soil and plants that with Steiner solution, while with $T_{8}$ was supplied organic matter and assimilable mineral by plants.

\section{Keywords}

Lycopersicon esculentum Mill., Macronutrients and Micronutrients, Beneficial Elements, Redox, Sustainability

${ }^{*}$ Corresponding author.

How to cite this paper: Delgado, J.M.P., Gutiérrez, E.Q., Ruvalcaba, L.P., Alcaraz, T.J.V., Valdés, T.D., Tafoya, F.A. and López, M.V. (2015) Yield of Tomato in Soil Treated with Compost, Amorphous Primary Minerals and Microorganisms. Open Access Library Journal, 2: e1865. http://dx.doi.org/10.4236/oalib.1101865 


\section{Introduction}

El suelo tiene un comportamiento dinámico, desde el origen de la vida se han extraído los nutrientes requeridos por la biota del suelo, que ha desarrollado procesos de mineralización, y que se emplea como mecanismos de protección y desarrollo. El suelo está compuesto por más de 1300 diferentes minerales primarios ricos en Si, Mg, $\mathrm{Ca}, \mathrm{K}, \mathrm{Fe}, \mathrm{P}, \mathrm{Zn}, \mathrm{Cu}, \mathrm{S}$, etc., los cuales son disueltos por la materia orgánica y los microorganismos para la nutrición de las plantas. Los suelos con alto contenido de minerales primarios (feldespatos, anfibolas y piroxenos, etc.) ofrecen una reserva considerable de nutrientes, de acuerdo con FitzPatrick [1], lo cual también se logra con roca pulverizada mediante un proceso práctico que reduce el consumo de energía y aumenta la disponibilidad de nutrientes al combinarse con microorganismos que solubilizan los minerales primarios amorfos, como lo menciona Stamford et al. [2].

Según la FAO [3], el suelo es uno de los ecosistemas más complejos de la naturaleza y uno de los hábitats más diversos de la tierra, alberga una infinidad de organismos diferentes que interactúan entre sí y contribuyen a los ciclos globales que hacen posible la vida. Los ciclos biogeoquímicos involucran a componentes geológicos (N, C, P, K, Ca, Mg, S, Na, Cl) y biológicos del ecosistema que involucran al agua, a los productores, consumidores y degradadores, según (Curtis y Sue [4], y son de fundamental importancia para mantener el equilibrio de la naturaleza debido a la participación y reutilización de los elementos en el ecosistema, como lo reportaron Fournier y Luis [5]).

De acuerdo con Bertrand et al. [6], todos los organismos vivos contribuyen a los ciclos biogeoquímicos, pero los microorganismos, debido a su gran abundancia, enormes capacidades metabólicas y el potencial de adaptación, desempeñan un papel clave en el funcionamiento y la evolución de los ciclos biogeoquímicos. En consecuencia, son fundamentales en la adaptación, resistencia y capacidad de recuperación de los ecosistemas.

Los componentes del entorno geológico son: 1) la atmósfera, constituida fundamentalmente por gases, que incluyen el vapor de agua, 2) la litosfera, la corteza sólida de la tierra, y 3) la hidrósfera, que comprende los océanos, lagos y ríos que cubren las tres cuartas partes de de la superficie terrestre. Los componentes biológicos de los ciclos biogeoquímicos incluyen a productores, consumidores y degradadores, así lo informaron Curtis y Barnes [7].

La óxido-reducción cambia drásticamente el comportamiento de los elementos en el suelo. En la naturaleza, la óxido-reducción siempre ocurre de manera simultánea y no quedan electrones libres, y su importancia radica en que es un proceso que origina las reacciones de energía dada por la transferencia de electrones. El oxígeno (O), carbono (C), nitrógeno (N), azufre (S), hierro (Fe) y manganeso (Mn), son los elementos involucrados en la capacidad redox y determinan en gran parte la génesis de suelos sulfatados ácidos, según Hinrich [8]. Iván et al. [9] descubrieron que la óxido-reducción con $\mathrm{mV}>400$ es un indicador de una fuerte oxidación que limita la productividad agrícola.

Miller y Augustine [10] mencionan que originalmente el término óxido-reducción fue aplicado a las reacciones en las cuales una sustancia se combinaba con el oxígeno, pero actualmente el concepto tiene un significado más amplio, ya que la reacción no requiere necesariamente la presencia de oxígeno, de tal manera que oxidación se define como la pérdida de electrones, o un aumento en el número de oxidación de un elemento hacía un valor más positivo, en tanto que reducción se refiere a la ganancia de electrones o una disminución en el número de oxidación hacia un valor menos positivo.

Actualmente para la producción agrícola se emplean insumos que provienen de la energía fósil, pero en cultivos como el tomate, las demandas de una fertilización con N son altas $\left(300-550 \mathrm{~kg} \cdot \mathrm{ha}^{-1}\right)$. Sin embargo, el N al igual que el P actúan como contaminantes en los flujos y fuentes de agua en Sinaloa, en el orden de 178,438 y 9890 t·año ${ }^{-1}$, respectivamente, y los sectores que más contribuyen en su contaminación son la agricultura y la ganadería, reportado por Paez [11]. Además, en el ámbito de la producción de alimentos vegetales, el tomate es la segunda hortaliza que más se produce en el mundo (aproximadamente 164 millones de t), correspondiendo a México el 2\% de la producción mundial, información de la FAO [3].

Rosset et al. [12] mencionan que actualmente es urgente la necesidad de impulsar un nuevo paradigma 
agrícola, para asegurar suficientes alimentos sanos y accesibles para la creciente población mundial, aunque tendrá que hacerse sobre la misma base de tierra arable, con menos petróleo, menos agua, nitrógeno y otros recursos, y dentro de un escenario de cambio climático y de incertidumbre económica y social. Asimismo, Espinoza [13] refiere que urge generar alternativas tecnológicas para mantener o recuperar la capacidad productiva de la tierra, así como preservar los recursos naturales y el ambiente.

Los fertilizantes orgánicos aportan grandes cantidades de materia orgánica, microorganismos y minerales, rehabilitando las características físicas, químicas y biológicas de los suelos, informado por (Martínez et al. [14]). Pero según Novella et al. [15], la agricultura moderna e intensiva debe tender a utilizar menos fertilizantes minerales y complementarse con el uso de biofertilizantes, debido a que los procesos microbiológicos implicados en su acción ofrecen ventajas de ser tecnologías que no contaminan el medio ambiente.

De acuerdo con Pocknee y Sumner [16], a partir de los tipos de materiales vegetales que se utilicen en la remediación de un suelo, por mineralización se liberan $\mathrm{N}$ y bases de cambio contenidas en compuestos orgánicos que afectan al $\mathrm{pH}$, de tal manera que el $\mathrm{N}$ contenido en los materiales vegetales originales provocaría un aumento inicial de $\mathrm{pH}$ asociado a la formación de $\mathrm{NH}_{4}^{+}$que consume protones. La posterior nitrificación del $\mathrm{NH}_{4}^{+}$en $\mathrm{NO}_{3}^{-}$resultaría en una disminución del $\mathrm{pH}$ debido a liberación de los protones a la solución del suelo.

Los objetivos de esta investigación consistieron en determinar la condición química inicial y final del suelo, después de haber sido tratado con composta, minerales primarios amorfos y microorganismos, así como la respuesta del tomate saladette (Lycopersicon esculentum Mill.) cultivado en esas condiciones durante un periodo de 150 días, bajo el supuesto de que si al suelo se le aporta materia orgánica, minerales primarios amorfos y microorganismos, se puede satisfacer la demanda de nutrientes del tomate en todo su ciclo vegetativo y productivo.

\section{Materiales y Métodos}

El trabajo experimental se realizó en un invernadero de la Facultad de Agronomía de la Universidad Autónoma de Sinaloa, ubicado en el kilómetro 17.5 de la carretera Culiacán-Eldorado, con coordenadas $24^{\circ} 48^{\prime} 28^{\prime \prime} \mathrm{N}$ y $107^{\circ} 24^{\prime} 30$ " O, en un suelo vertisol crómico con $\mathrm{pH}=7.3, \mathrm{CE}=0.92 \mathrm{dS} \cdot \mathrm{m}^{-1}, \mathrm{MO}=0.86 \%, \mathrm{RAS}=1.1, \mathrm{~N}=147$ $\mathrm{kg} \cdot \mathrm{ha}^{-1}$ y P $=28.7 \mathrm{~kg} \cdot \mathrm{ha}^{-1}$. De acuerdo con García [17], el clima es semiseco, muy cálido, extremoso, con lluvias en verano, con temperatura media anual de $25.9^{\circ} \mathrm{C}$ y precipitación media anual de $772 \mathrm{~mm}$.

Se utilizó tomate saladette (Lycopersicon esculentum Mill.) cv "Moctezuma" con hábito de crecimiento indeterminado, el cual se cultivó en surcos separados a $1.6 \mathrm{~m}$ y una densidad de población de 25,000 plantas·ha ${ }^{-1}$. El trasplante se realizó el 12 de diciembre de 2011 y las plantas se manejaron a un tallo, en diseño de bloques completos al azar con cuatro repeticiones y los tratamientos indicados en la Tabla 1. La solución Steiner se definió con base en lo recomendado por Steiner [18], mientras que los otros tratamientos se construyeron teniendo en cuenta que la composta contiene de 1\% - 3\% de N, con mineralización del 18\%, información de Egbahall [19],

Tabla 1. Tratamientos utilizados para la producción de tomate saladette en invernadero. Ciclo agrícola 2011-2012. Culiacán, Sinaloa (México).

\begin{tabular}{|c|c|c|}
\hline Tratamientos y control & Abrev. & Sustancias/mezclas \\
\hline Control (Tratamiento 1) & $\mathrm{T}_{1}$ & Solución Steiner 100 \% \\
\hline Tratamiento 2 & $\mathrm{~T}_{2}$ & $15 \mathrm{Mg} \cdot \mathrm{ha}^{-1}$ composta $+3 \mathrm{Mg} \cdot \mathrm{ha}^{-1} \mathrm{MPA}$ \\
\hline Tratamiento 3 & $\mathrm{~T}_{3}$ & $15 \mathrm{Mg} \cdot \mathrm{ha}^{-1}$ composta $+3 \mathrm{Mg} \cdot \mathrm{ha}^{-1} \mathrm{MPA}+2 \mathrm{~L} \cdot \mathrm{ha}^{-1}$ de $\mathrm{M}$ \\
\hline Tratamiento 4 & $\mathrm{~T}_{4}$ & $15 \mathrm{Mg} \cdot \mathrm{ha}^{-1}$ composta $+6 \mathrm{Mg} \cdot \mathrm{ha}^{-1} \mathrm{MPA}$ \\
\hline Tratamiento 5 & $\mathrm{~T}_{5}$ & $15 \mathrm{Mg} \cdot \mathrm{ha}^{-1}$ composta $+6 \mathrm{Mg} \cdot \mathrm{ha}^{-1} \mathrm{MPA}+2 \mathrm{~L} \cdot \mathrm{ha}^{-1}$ de $\mathrm{M}$ \\
\hline Tratamiento 6 & $\mathrm{~T}_{6}$ & $25 \mathrm{Mg} \cdot \mathrm{ha}^{-1}$ composta $+3 \mathrm{Mg} \cdot \mathrm{ha}^{-1} \mathrm{MPA}$ \\
\hline Tratamiento 7 & $\mathrm{~T}_{7}$ & $25 \mathrm{Mg} \cdot \mathrm{ha}^{-1}$ composta $+3 \mathrm{Mg} \cdot \mathrm{ha}^{-1} \mathrm{MPA}+2 \mathrm{~L} \cdot \mathrm{ha}^{-1}$ de $\mathrm{M}$ \\
\hline Tratamiento 8 & $\mathrm{~T}_{8}$ & $25 \mathrm{Mg} \cdot \mathrm{ha}^{-1}$ composta $+6 \mathrm{Mg} \cdot \mathrm{ha}^{-1} \mathrm{MPA}$ \\
\hline Tratamiento 9 & $\mathrm{~T}_{9}$ & $25 \mathrm{Mg} \cdot \mathrm{ha}^{-1}$ composta $+6 \mathrm{Mg} \cdot \mathrm{ha}^{-1} \mathrm{MPA}+2 \mathrm{~L} \cdot \mathrm{ha}^{-1}$ de $\mathrm{M}$ \\
\hline
\end{tabular}

MPA = Minerales Primarios Amorfos; $\mathrm{M}=$ Microorganismos. 
así como lo recomendado por los distribuidores de minerales primarios amorfos (MPA) y de microorganismos para definir el consorcio microbiano (Tabla 2). En la parcela testigo, la solución Steiner se aplicó desde el trasplante hasta el fin de cosecha a través de un sistema de riego por goteo con doble manguera por cada surco con goteros cada $30 \mathrm{~cm}$, en tanto que en las parcelas donde se asignaron los otros tratamientos sólo se aplicó agua con el mismo sistema, y donde se planeó la aplicación de microorganismos, éstos se aplicaron cada ocho días a través de dicho sistema de riego.

La composta bocashi (rastrojo de maíz, estiércol de bovino, salvado de arroz, cascarilla de arroz, melaza, levadura de pan, carbón vegetal triturado, tierra de aluvión y agua) y los MPA se aplicaron al suelo un mes antes del trasplante (adt), manteniéndose a capacidad de campo durante 30 días, posteriormente el suelo se removió y mezcló adt.

Se realizó un muestreo de suelo a una profundidad de 0 - $30 \mathrm{~cm}$ antes del trasplante y otro al final del cultivo, y aplicando la metodología de Quero [20], las muestras de suelo se secaron a $40^{\circ} \mathrm{C}$ y se molieron a un tamaño de partícula de $1.0 \mu \mathrm{m}$, y se compactaron con prensa hidráulica para obtener una pastilla con una superficie homogénea y liza. Posteriormente, se leyeron en un equipo compuesto por un espectrómetro de rayos X, acoplados a un microscopio electrónico de barrido (MEB) marca Jeol modelo JSM-6390 LV LGS.

El espectrómetro de energía dispersiva de rayos X modelo LK-IE250 OXFORD INCA ENERGY 250 permite, por emisión de un haz de electrones primarios que inciden en la muestra y generan electrones secundarios que son colectados por un detector de electrones, la cuantificación total de elementos químicos que se encuentran en una concentración superior al $0.1 \%$ del peso seco de la muestra de suelo, con lo que se analizó el contenido de C, O, N, P, K, Ca, Mg, S, Zn, Cl, Mn, Na, Fe, Ti, Al y Si. Asimismo, el pH, CE, CIC y el potencial óxido-reducción (ORP) presente en dichos sitios.

\section{Resultados y Discusión}

Los porcentajes de $\mathrm{C}$ que se observan en la Tabla 3 indican que antes del trasplante se aportó menos energía con $\mathrm{T}_{8}$ y donde posteriormente se aplicaría solución Steiner para el crecimiento y desarrollo de las plantas de tomate, mientras que con el resto de tratamientos la aportación fue mayor, ya que los altos valores de C que se detectaron, comparados con los porcentajes encontrados donde se aplicaron solución Steiner y $\mathrm{T}_{8}$, quizás fueron producto de la mineralización de la materia orgánica por los microorganismos adicionados al suelo con la composta en conjunto con los ya existentes en dicho recurso natural. Sin embargo, posterior a la cosecha el C ya no fue-

Tabla 2. Características físico-químicas de la composta y minerales primarios amorfos y concentraciones utilizadas de las especies del consorcio microbiano.

\begin{tabular}{|c|c|c|c|c|c|}
\hline Característica & Composta & Característica & Minerales primarios amorfos & Consorcio microbiano & Concentración \\
\hline $\mathrm{pH}$ & 9.30 & $\mathrm{Ph}$ & 8.9 & Bacillus subtilis & $1 \times 10^{18} \mathrm{ufc} \cdot \mathrm{ml}^{-1}$ \\
\hline $\mathrm{CE}\left(\mathrm{dS} \cdot \mathrm{m}^{-1}\right)$ & 12.0 & $\mathrm{CE}\left(\mathrm{dS} \cdot \mathrm{m}^{-1}\right)$ & 4.5 & Trichoderma harzianum & $2 \times 10^{8}$ ufc $\cdot \mathrm{g}^{-1}$ \\
\hline MO (\%) & 10.78 & $\mathrm{SiO}_{2}(\%)$ & 34 & Azotobacters pp & $1 \times 10^{6} \mathrm{ufc} \cdot \mathrm{g}^{-1}$ \\
\hline N-total (\%) & 0.78 & $\mathrm{P}_{2} \mathrm{O}_{5}(\%)$ & 9.6 & Azozpirillum brasilense & $1 \times 10^{6}$ ufc $\cdot \mathrm{g}^{-1}$ \\
\hline $\mathrm{P}_{2} \mathrm{O}_{5}(\%)$ & 0.34 & $\mathrm{~K}_{2} \mathrm{O}(\%)$ & 2.5 & Glomusin traradices & 20 esporas $\cdot g^{-1}$ \\
\hline $\mathrm{K}_{2} \mathrm{O}(\%)$ & 1.90 & $\mathrm{CaO}(\%)$ & 13 & & \\
\hline $\mathrm{Ca}(\%)$ & 1.48 & MgO (\%) & 2.0 & & \\
\hline $\operatorname{Mg}(\%)$ & 0.93 & $\mathrm{Fe}\left(\mathrm{mg} \cdot \mathrm{kg}^{-1}\right)$ & 18 & & \\
\hline $\mathrm{Fe}\left(\mathrm{mg} \cdot \mathrm{kg}^{-1}\right)$ & 1.9 & $\mathrm{Zn}\left(\mathrm{mg} \cdot \mathrm{kg}^{-1}\right)$ & 16 & & \\
\hline $\mathrm{Cu}\left(\mathrm{mg} \cdot \mathrm{kg}^{-1}\right)$ & 16 & $\operatorname{Mn}\left(\mathrm{mg} \cdot \mathrm{kg}^{-1}\right)$ & 5 & & \\
\hline $\mathrm{Zn}\left(\mathrm{mg} \cdot \mathrm{kg}^{-1}\right)$ & 65 & & & & \\
\hline $\mathrm{Mn}\left(\mathrm{mg} \cdot \mathrm{kg}^{-1}\right)$ & 390 & & & & \\
\hline $\mathrm{B}\left(\mathrm{mg} \cdot \mathrm{kg}^{-1}\right)$ & 120 & & & & \\
\hline
\end{tabular}


Tabla 3. Contenido porcentual de macronutrientes del suelo antes del trasplante y después de la cosecha. Ciclo agrícola 2011-2012, Culiacán, Sinaloa, México.

\begin{tabular}{ccccccccc}
\hline \multirow{2}{*}{ Trat. } & \multicolumn{2}{c}{$\mathrm{C}(\%)$} & \multicolumn{2}{c}{$\mathrm{O}(\%)$} & \multicolumn{2}{c}{$\mathrm{N}(\%)$} & \multicolumn{2}{c}{$\mathrm{P}(\%)$} \\
\cline { 2 - 9 } & Antes & Después & Antes & Después & Antes & Después & Antes & Después \\
\hline $\mathrm{T}_{1}$ & $3.183 \mathrm{~b}$ & $0.000 \mathrm{c}$ & $56.010 \mathrm{a}$ & $56.125 \mathrm{a}$ & $0.243 \mathrm{a}$ & $3.372 \mathrm{abc}$ & $0.183 \mathrm{ab}$ & $0.350 \mathrm{a}$ \\
$\mathrm{T}_{2}$ & $9.313 \mathrm{a}$ & $7.220 \mathrm{a}$ & $52.292 \mathrm{c}$ & $53.075 \mathrm{c}$ & $0.206 \mathrm{ab}$ & $3.882 \mathrm{ab}$ & $0.290 \mathrm{ab}$ & $0.242 \mathrm{a}$ \\
$\mathrm{T}_{3}$ & $7.465 \mathrm{a}$ & $5.368 \mathrm{ab}$ & $53.390 \mathrm{bc}$ & $54.485 \mathrm{abc}$ & $0.183 \mathrm{ab}$ & $4.070 \mathrm{ab}$ & $0.363 \mathrm{ab}$ & $0.240 \mathrm{a}$ \\
$\mathrm{T}_{4}$ & $7.423 \mathrm{a}$ & $6.985 \mathrm{a}$ & $53.902 \mathrm{~b}$ & $53.382 \mathrm{c}$ & $0.217 \mathrm{a}$ & $3.415 \mathrm{abc}$ & $0.366 \mathrm{ab}$ & $0.297 \mathrm{a}$ \\
$\mathrm{T}_{5}$ & $9.713 \mathrm{a}$ & $8.165 \mathrm{a}$ & $52.432 \mathrm{bc}$ & $53.902 \mathrm{bc}$ & $0.133 \mathrm{~b}$ & $4.232 \mathrm{a}$ & $0.243 \mathrm{ab}$ & $0.277 \mathrm{a}$ \\
$\mathrm{T}_{6}$ & $8.418 \mathrm{a}$ & $6.170 \mathrm{ab}$ & $53.130 \mathrm{bc}$ & $54.492 \mathrm{abc}$ & $0.240 \mathrm{a}$ & $2.522 \mathrm{c}$ & $0.366 \mathrm{ab}$ & $0.347 \mathrm{a}$ \\
$\mathrm{T}_{7}$ & $9.613 \mathrm{a}$ & $4.850 \mathrm{ab}$ & $52.677 \mathrm{bc}$ & $54.275 \mathrm{abc}$ & $0.206 \mathrm{ab}$ & $2.385 \mathrm{c}$ & $0.426 \mathrm{a}$ & $0.235 \mathrm{a}$ \\
$\mathrm{T}_{8}$ & $3.183 \mathrm{~b}$ & $3.020 \mathrm{bc}$ & $55.860 \mathrm{a}$ & $55.527 \mathrm{ab}$ & $0.247 \mathrm{a}$ & $3.157 \mathrm{bc}$ & $0.110 \mathrm{~b}$ & $0.207 \mathrm{a}$ \\
$\mathrm{T}_{9}$ & $9.870 \mathrm{a}$ & $6.925 \mathrm{a}$ & $52.377 \mathrm{bc}$ & $54.032 \mathrm{bc}$ & $0.243 \mathrm{a}$ & $3.185 \mathrm{abc}$ & $0.360 \mathrm{ab}$ & $0.307 \mathrm{a}$ \\
$\mathrm{DMSH}$ & 3.934 & 3.753 & 1.569 & 1.902 & 0.078 & 1.069 & 0.309 & 0.177 \\
\hline
\end{tabular}

Medias con diferente letra en la columna, son estadísticamente diferentes (Tukey, 0.05). Continúa.

\begin{tabular}{|c|c|c|c|c|c|c|c|c|}
\hline \multirow{2}{*}{ Trat. } & \multicolumn{2}{|c|}{ K (\%) } & \multicolumn{2}{|c|}{ Ca (\%) } & \multicolumn{2}{|c|}{ Mg (\%) } & \multicolumn{2}{|c|}{ S (\%) } \\
\hline & Antes & Después & Antes & Después & Antes & Después & Antes & Después \\
\hline $\mathrm{T}_{1}$ & $1.053 \mathrm{a}$ & 0.946 a & $2.033 \mathrm{a}$ & $2.305 \mathrm{a}$ & $1.683 \mathrm{a}$ & 1.820 a & $0.226 \mathrm{a}$ & 0.0 \\
\hline $\mathrm{T}_{2}$ & $1.263 \mathrm{a}$ & $1.146 \mathrm{a}$ & $2.080 \mathrm{a}$ & $2.065 \mathrm{~b}$ & $1.416 \mathrm{ab}$ & $1.393 \mathrm{bcd}$ & $0.010 \mathrm{a}$ & 0.0 \\
\hline $\mathrm{T}_{3}$ & $1.110 \mathrm{a}$ & $1.150 \mathrm{a}$ & $2.360 \mathrm{a}$ & $1.990 \mathrm{~b}$ & $1.430 \mathrm{ab}$ & $1.447 \mathrm{bcd}$ & $0.027 \mathrm{a}$ & 0.0 \\
\hline $\mathrm{T}_{4}$ & $1.216 \mathrm{a}$ & $1.133 \mathrm{a}$ & $2.260 \mathrm{a}$ & $2.085 a b$ & $1.417 \mathrm{ab}$ & $1.370 \mathrm{~cd}$ & 0.033 a & 0.0 \\
\hline $\mathrm{T}_{5}$ & $1.170 \mathrm{a}$ & $1.007 \mathrm{a}$ & $2.093 \mathrm{a}$ & $2.020 \mathrm{~b}$ & $1.440 \mathrm{ab}$ & $1.256 \mathrm{~d}$ & 0.033 a & 0.0 \\
\hline $\mathrm{T}_{6}$ & 0.990 a & $1.063 \mathrm{a}$ & $2.233 \mathrm{a}$ & $2.147 \mathrm{ab}$ & $1.383 a b$ & 1.543 bc & $0.077 \mathrm{a}$ & 0.0 \\
\hline $\mathrm{T}_{7}$ & $1.193 \mathrm{a}$ & $1.190 \mathrm{a}$ & $2.263 \mathrm{a}$ & $2.110 \mathrm{ab}$ & $1.363 \mathrm{~b}$ & $1.566 \mathrm{abc}$ & 0.067 a & 0.0 \\
\hline $\mathrm{T}_{8}$ & 0.946 a & 0.950 a & 1.945 a & $1.987 \mathrm{~b}$ & $1.597 \mathrm{ab}$ & $1.606 \mathrm{abc}$ & 0.203 a & 0.0 \\
\hline $\mathrm{T}_{9}$ & $1.090 \mathrm{a}$ & 0.950 a & $2.173 \mathrm{a}$ & $2.025 \mathrm{~b}$ & $1.370 \mathrm{ab}$ & $1.630 \mathrm{ab}$ & $0.200 \mathrm{a}$ & 0.0 \\
\hline DMSH & 0.280 & 0.274 & 0.642 & 0.385 & 0.213 & 0.222 & 0.251 & 0.0 \\
\hline
\end{tabular}

Medias con diferente letra en la columna, son estadísticamente diferentes (Tukey, 0.05).

detectado donde se aplicó solución Steiner, pero donde se incorporó $\mathrm{T}_{8}$ dicho elemento sólo disminuyó ligeramente (5.1\%) en relación a lo estimado en las mismas parcelas antes del trasplante. Lo anterior coincide con lo reportado por Martínez et al. [14], ya que éllos refieren que con la mineralización de la materia orgánica del suelo (MOS) se liberan diversos nutrientes para las plantas, muchos de los cuales son aportados en forma deficitaria por los minerales del suelo, y que el C orgánico del suelo (COS) interviene en las propiedades biológicas, básicamente actuando como fuente energética para los organismos heterótrofos del suelo. Asimismo, que el COS, a través de los efectos en las propiedades físicas, químicas y biológicas del suelo ha resultado ser el principal determinante de su productividad. Además, estos resultados confirman lo declarado por la FAO [21] y lo descubierto por Craswell y Lefroy [22], en el sentido de que la agricultura de conservación, abonos verdes, cultivos de cobertura, compostas y abonos orgánicos son una fuente importante para la captura de carbono.

La cantidad de oxígeno antes del trasplante fue estadísticamente superior en un rango de $0.3 \%$ a $7.1 \%$ en las parcelas donde se aplicó solamente solución Steiner (Tabla 3) en comparación a lo encontrado donde se aplicaron los demás tratamientos; asimismo, después de la cosecha, pero el rango de incrementos osciló de $1.1 \%$ a 
5.7\%, de tal manera que como lo señalan Singer y Munns [23], esto significa que el oxígeno no fue un factor limitante en el suelo para que la descomposición de la materia orgánica se produjera en parte por respiración, y que de acuerdo al estado oxidativo del suelo el $\mathrm{C}$ mineralizado pudo adoptar las formas oxidada $\left(\mathrm{CO}_{2}\right)$ o reducida $\left(\mathrm{CH}_{4}\right)$.

A diferencia del C y O, los porcentajes de $\mathrm{N}$ que se encontraron antes del trasplante en todas las parcelas fueron muy variables, de tal manera que las mayores cantidades se obtuvieron en aquellas áreas tratadas con solución Steiner, $\mathrm{T}_{4}, \mathrm{~T}_{6}, \mathrm{~T}_{8}$ y $\mathrm{T}_{9}$, con los respectivos incrementos de $82.7 \%, 63.1 \%$, 80.4\% y 82.7\% en relación a lo estimado en la unidades experimentales tratadas con $T_{5}$. No obstante, los porcentajes fueron mucho más elevados después de la cosecha, a tal grado que ya no se encontraron valores menores a la unidad porcentual, sino de 2.4 a 4.2 unidades; sin embargo, con $\mathrm{T}_{3}$ y $\mathrm{T}_{5}$ los porcentajes se incrementaron $20.3 \%$ y $25.5 \%$ con respecto a lo encontrado donde se trató con solución Steiner, aunque los valores no fueron estadísticamente diferentes; en cambio, con $\mathrm{T}_{6} \mathrm{y} \mathrm{T}_{7}$ los porcentajes fueron 25.2\% y 29.3\% inferiores a lo alcanzado con solución Steiner. Según Pocknee y Sumner [16], estos valores de $\mathrm{N}$ antes del trasplante y después de la cosecha podrían explicarse por la liberación por mineralización de las bases de cambio contenidas en los compuestos orgánicos y por el contenido inicial de $\mathrm{N}$ en los materiales vegetales utilizados en la composta.

En la concentración de P también se encontraron cantidades muy variables antes del trasplante (Tabla 3), de tal forma que el mayor incremento (132.8\%) se dio con $\mathrm{T}_{7}$ comparado a lo obtenido con solución Steiner; mientras que con $\mathrm{T}_{8}$ disminuyó $39.9 \%$. Después de la cosecha, el mayor contenido de $\mathrm{P}$ se obtuvo donde se aplicó solución Steiner, lo cual superó a los demás valores en un rango de $0.9 \%$ a $69.1 \%$, sin que se notaran diferencias estadísticas entre las porcentajes. Lo cual de acuerdo con Oades et al. [24] y Krull et al. [25], fue favorecido por la unión de iones con ligandos provenientes de la materia orgánica aplicada, lo que a su vez mejoró la fertilidad del suelo con la disponibilidad de P por bloqueo de potenciales sitios de reacción con Fe, Al y Ca. Máxime que los valores fueron superiores al rango de $0.01 \%-0.15 \%$ reportado por Cajuste [26], así como al de $0.02 \%-0.08 \%$ reportado por Fassbender y Bornemiza [27].

En lo correspondiente al K se observó que este elemento estuvo presente en el suelo de una forma variable, pero sin diferencia estadística entre los porcentajes antes del trasplante y después de la cosecha (Tabla 3); en cuanto al Ca ocurrió algo parecido antes del trasplante, pero después de la cosecha los porcentajes variaron estadísticamente y el mayor valor porcentual se encontró donde se aplicó solución Steiner, lo que a su vez superó en $11.6 \%, 15.8 \%, 14.1 \%, 16.0 \%$ y $13.8 \%$ a los valores obtenidos donde se aplicaron $\mathrm{T}_{2}, \mathrm{~T}_{3}, \mathrm{~T}_{5}, \mathrm{~T}_{8}$ y $\mathrm{T}_{9}$, respectivamente. El Mg varió estadísticamente antes del trasplante, pero el porcentaje donde se aplicaría solución Steiner sólo se incrementó en $23.5 \%$ comparado con el valor porcentual estimado donde el suelo fue tratado con $\mathrm{T}_{7}$, mientras que posterior a la cosecha el porcentaje en las mismas parcelas superó en $30.6 \%, 25.8 \%$, 32.8\%, 44.9\% y $17.9 \%$ a los valores porcentuales encontrados donde se incorporaron los respectivos $\mathrm{T}_{2}, \mathrm{~T}_{3}, \mathrm{~T}_{4}, \mathrm{~T}_{5}, \mathrm{y} \mathrm{T}_{6}$.

El azufre no varió estadísticamente antes del trasplante, pero los porcentajes más elevados se obtuvieron en las parcelas donde se aplicaría solución Steiner y donde se aplicaron $\mathrm{T}_{8}$ y $\mathrm{T}_{9}$, siendo con mucho superior a lo estimado en las demás parcelas; sin embargo, después de la cosecha este elemento ya no se detectó en las muestras de suelo. Estos resultados indican que después de la cosecha el contenido de $\mathrm{S}$ en el suelo de las parcelas experimentales disminuyó por debajo del $0.1 \%$ que recomienda Takkar [28], mientras que el contenido de $\mathrm{K}$ estuvo dentro del rango de $0.5 \%$ - 2.5\% informado por Tisdale et al. [29], en tanto que los de Ca fueron muy superiores al rango de $0.1 \%$ - $0.3 \%$ referido por Prassad y Power [30] y al 0.12\% que Nuez [31] recomienda para que en el suelo no se presenten deficiencias en tomate. La deficiencia de $\mathrm{S}$ detectada después de la cosecha quizás se debió a que el suelo utilizado en esta investigación recibió, en ciclos agrícolas anteriores, escasas aplicaciones de materia orgánica, lo que inevitablemente condujo a la deficiencia de azufre, según Messick et al. [32]. De igual manera sucedió con el Mg, ya que según Fassbender y Bornemiza [27] el Mg en suelos no calcáreos varía de 0.1 a $1 \%$.

El contenido de $\mathrm{Zn}$ antes del trasplante se indica en la Tabla 4, donde se puede notar que el mayor porcentaje se obtuvo con $\mathrm{T}_{2}$, lo que a su vez superó en 2.85 veces a lo encontrado donde se aplicaría solución Steiner, sin que entre ambos valores hubiera diferencia estadística; sin embargo, donde se aplicaron $\mathrm{T}_{5} \mathrm{y} \mathrm{T}_{8}$ el equipo de rayos x no detectó a este elemento. Después de la cosecha se observó que los valores disminuyeron 46.1\%, $76.0 \%, 63.6 \%, 25.6 \%, 100 \%$ y $100 \%$, en las parcelas donde se aplicaron $\mathrm{T}_{1}, \mathrm{~T}_{2}, \mathrm{~T}_{3}, \mathrm{~T}_{4}, \mathrm{~T}_{7}$ y $\mathrm{T}_{9}$, respectivamente, en tanto que con $\mathrm{T}_{6} \mathrm{y}_{\mathrm{T}}$ los valores no variaron, pero con $\mathrm{T}_{5}$ este elemento se detectó en el porcentaje que se indica en la tabla. No obstante, el contenido de Zn encontrado antes del trasplante en esta investigación fue muy inferior a lo recomendado por Prasad y Power [30], ya que éllos refieren que el contenido de $\mathrm{Zn}$ en el suelo debe- 
Tabla 4. Contenido de micronutrientes y elementos beneficiosos del suelo antes del trasplante y después de la cosecha. Ciclo agrícola 2011-2012, Culiacán, Sinaloa. México.

\begin{tabular}{cccccccccc}
\hline & \multicolumn{2}{c}{$Z n(\%)$} & \multicolumn{2}{c}{$\mathrm{Cl}(\%)$} & \multicolumn{2}{c}{ Mn (\%) } & \multicolumn{2}{c}{ Na (\%) } \\
\cline { 2 - 9 } Trat. & Antes & Después & Antes & Después & Antes & Después & Antes & Después \\
\cline { 2 - 9 } $\mathrm{T}_{1}$ & $0.013 \mathrm{abc}$ & $0.007 \mathrm{a}$ & $0.0 \mathrm{a}$ & 0.0 & $0.083 \mathrm{~b}$ & $0.096 \mathrm{a}$ & $0.343 \mathrm{~b}$ & $0.096 \mathrm{a}$ \\
$\mathrm{T}_{2}$ & $0.050 \mathrm{a}$ & $0.012 \mathrm{a}$ & $0.0 \mathrm{a}$ & 0.0 & $0.100 \mathrm{a}$ & $0.103 \mathrm{a}$ & $0.413 \mathrm{ab}$ & $0.103 \mathrm{a}$ \\
$\mathrm{T}_{3}$ & $0.033 \mathrm{abc}$ & $0.012 \mathrm{a}$ & $0.0 \mathrm{a}$ & 0.0 & $0.093 \mathrm{a}$ & $0.096 \mathrm{a}$ & $0.446 \mathrm{ab}$ & $0.096 \mathrm{a}$ \\
$\mathrm{T}_{4}$ & $0.043 \mathrm{ab}$ & $0.032 \mathrm{a}$ & $0.0 \mathrm{a}$ & 0.0 & $0.100 \mathrm{a}$ & $0.076 \mathrm{a}$ & $0.440 \mathrm{ab}$ & $0.076 \mathrm{a}$ \\
$\mathrm{T}_{5}$ & $0.000 \mathrm{c}$ & $0.025 \mathrm{a}$ & $0.0 \mathrm{a}$ & 0.0 & $0.100 \mathrm{a}$ & $0.080 \mathrm{a}$ & $0.516 \mathrm{a}$ & $0.080 \mathrm{a}$ \\
$\mathrm{T}_{6}$ & $0.010 \mathrm{bc}$ & $0.010 \mathrm{a}$ & $0.0 \mathrm{a}$ & 0.0 & $0.106 \mathrm{a}$ & $0.086 \mathrm{a}$ & $0.433 \mathrm{ab}$ & $0.086 \mathrm{a}$ \\
$\mathrm{T}_{7}$ & $0.017 \mathrm{abc}$ & $0.000 \mathrm{a}$ & $0.0 \mathrm{a}$ & 0.0 & $0.093 \mathrm{a}$ & $0.106 \mathrm{a}$ & $0.453 \mathrm{ab}$ & $0.106 \mathrm{a}$ \\
$\mathrm{T}_{8}$ & $0.000 \mathrm{c}$ & $0.000 \mathrm{a}$ & $0.032 \mathrm{a}$ & 0.0 & $0.110 \mathrm{a}$ & $0.096 \mathrm{a}$ & $0.386 \mathrm{ab}$ & $0.096 \mathrm{a}$ \\
$\mathrm{T}_{9}$ & $0.013 \mathrm{abc}$ & $0.000 \mathrm{a}$ & $0.0 \mathrm{a}$ & 0.0 & $0.093 \mathrm{a}$ & $0.100 \mathrm{a}$ & $0.413 \mathrm{ab}$ & $0.100 \mathrm{a}$ \\
$\mathrm{DMSH}$ & 0.050 & 0.050 & 0.038 & 0.0 & 0.027 & 0.046 & 0.155 & 0.289 \\
\hline
\end{tabular}

Medias con diferente letra en la columna, son estadísticamente diferentes (Tukey, 0.05). Continúa.

\begin{tabular}{|c|c|c|c|c|c|c|c|c|}
\hline \multirow{2}{*}{ Trat. } & \multicolumn{2}{|c|}{$\mathrm{Fe}(\%)$} & \multicolumn{2}{|c|}{ Ti (\%) } & \multicolumn{2}{|c|}{$\mathrm{Al}(\%)$} & \multicolumn{2}{|c|}{ Si (\%) } \\
\hline & Antes & Después & Antes & Después & Antes & Después & Antes & Después \\
\hline $\mathrm{T}_{1}$ & $4.912 \mathrm{ab}$ & $5.357 \mathrm{a}$ & $0.180 \mathrm{ab}$ & $0.192 \mathrm{a}$ & 6.992 a & $7.422 \mathrm{a}$ & $20.212 \mathrm{a}$ & $22.112 \mathrm{a}$ \\
\hline $\mathrm{T}_{2}$ & $4.692 \mathrm{ab}$ & $4.722 \mathrm{ab}$ & $0.165 \mathrm{ab}$ & $0.177 \mathrm{a}$ & 5.995 c & 6.282 bc & $19.382 \mathrm{a}$ & $19.477 \mathrm{~cd}$ \\
\hline $\mathrm{T}_{3}$ & $4.830 \mathrm{ab}$ & $4.810 \mathrm{ab}$ & $0.155 \mathrm{ab}$ & $0.187 \mathrm{a}$ & 6.34 abc & 6.365 bc & $19.622 \mathrm{a}$ & $19.625 \mathrm{~cd}$ \\
\hline $\mathrm{T}_{4}$ & $4.510 \mathrm{~b}$ & $4.932 \mathrm{ab}$ & $0.150 \mathrm{~b}$ & $0.180 \mathrm{a}$ & $6.210 \mathrm{bc}$ & 6.405 bc & $19.212 \mathrm{a}$ & $19.635 \mathrm{~cd}$ \\
\hline $\mathrm{T}_{5}$ & $4.672 \mathrm{ab}$ & $4.350 \mathrm{~b}$ & $0.162 \mathrm{ab}$ & $0.175 \mathrm{a}$ & 6.110 bc & 5.905 c & $19.012 \mathrm{a}$ & $18.390 \mathrm{~d}$ \\
\hline $\mathrm{T}_{6}$ & $4.620 \mathrm{ab}$ & $4.945 \mathrm{ab}$ & $0.165 \mathrm{ab}$ & 0.185 a & 6.122 bc & 6.342 bc & 18.917 a & $20.025 \mathrm{~cd}$ \\
\hline $\mathrm{T}_{7}$ & $4.752 \mathrm{ab}$ & $5.165 \mathrm{a}$ & $0.165 \mathrm{ab}$ & $0.185 \mathrm{a}$ & 5.840 c & $6.730 \mathrm{abc}$ & $18.457 \mathrm{a}$ & 21.135 abc \\
\hline $\mathrm{T}_{8}$ & $5.280 \mathrm{a}$ & 5.295 a & $0.182 \mathrm{a}$ & 0.195 a & $6.785 \mathrm{ab}$ & $7.030 \mathrm{ab}$ & $19.460 \mathrm{a}$ & $21.535 \mathrm{ab}$ \\
\hline $\mathrm{T}_{9}$ & $4.695 \mathrm{ab}$ & $4.895 \mathrm{ab}$ & $0.160 \mathrm{ab}$ & $0.175 \mathrm{a}$ & 5.830 c & $6.260 \mathrm{bc}$ & $18.850 \mathrm{a}$ & $19.362 \mathrm{~cd}$ \\
\hline DMSH & 0.706 & 0.772 & 0.030 & 0.026 & 0.737 & 0.838 & 2.171 & 1.874 \\
\hline
\end{tabular}

Medias con diferente letra en la columna, son estadísticamente diferentes (Tukey, 0.05).

ser de aproximadamente $0.5-70 \mathrm{mg} \cdot \mathrm{Mg}^{-1}$, puesto que las plantas de tomate requieren este nutriente para la síntesis de del ácido B-indolacético, una de las hormonas de crecimiento, que de acuerdo con Navarro [33], aumenta o disminuye de forma paralela con el contenido de triptófano que es uno de sus precursores, y que de acuerdo con Vallee [34] dicho aminoácido debe ser sintetizado con ayuda de una de las 80 enzimas que contienen $\mathrm{Zn}$.

El Cl fue uno de los elementos que el sistema de rayos $\times$ sólo detectó antes del trasplante en las parcelas que recibieron tratamiento con $\mathrm{T}_{8}$ (Tabla 4), lo que significa que los porcentajes de $\mathrm{Cl}$ encontrados en esta investigación antes del trasplante no fueron suficientes para satisfacer las necesidades de las plantas de tomate, ya que Navarro [33] indica que los requerimientos fisiológicos son de 5 ppm, toda vez que de acuerdo con Navarro y Navarro [35] el contenido medio de $\mathrm{Cl}$ en las plantas es de $2-20 \mathrm{mg} \cdot \mathrm{g}^{-1}$ en peso seco, aunque también refieren que para un crecimiento óptimo de la mayoría de las plantas, el contenido debe estar en un rango de 0.2 - 0.4 $\mathrm{mg} \cdot \mathrm{g}^{-1}$ peso seco. Sin embargo, esta cantidad muy baja de $\mathrm{Cl}$ en las parcelas con $\mathrm{T}_{8}$ debió tener consecuencias en el rendimiento de tomate, porque justamente con este tratamiento fue con el que se obtuvo la mayor producción total. 
El Mn estuvo presente con diferencias estadísticas antes del trasplante, con incrementos que fueron desde 12.0\% - 32.5\% con todos los tratamientos que incluyeron composta, con respecto al valor obtenido con $\mathrm{T}_{1}$; no obstante, después de la cosecha ya no se observaron incrementos con diferencias estadísticas, por lo que de acuerdo con Salisbury y Ross [36] las plantas de tomate tuvieron suficiente Mn para la activación de enzimas importantes en la respiración, en reacciones del metabolismo del nitrógeno y la fotosíntesis, así como para el metabolismo de la hormona ácido indolacético, aunque el papel más importante del manganeso en la fotosíntesis quizás fue en la secuencia de reacciones mediante para derivar electrones del agua y liberar oxígeno. De no haber estado el Mn en cantidades suficientes, en las plantas se hubieran observado síntomas de deficiencia.

El Na fue otro elemento que antes del trasplante se detectó con incrementos de $12.5 \%$ - 50.4\% donde se incorporaron tratamientos que contuvieron composta, comparado con el porcentaje estimado donde se aplicaría $\mathrm{T}_{1}$, aunque sólo hubo diferencia estadística con respecto al valor obtenido con $\mathrm{T}_{5}$; después de la cosecha ya no se observaron incrementos en relación al valor observado donde se aplicaría $\mathrm{T}_{1}$, pero entre los respectivos pares de valores hubo disminuciones de $72.0 \%, 75.1 \%$, 78.5\%, 82.7\%, 84.5\%, 80.1\%, 76.6\%, 75.1\% y $75.8 \%$ de dicho elemento, lo que conllevó a que las sales no se acumularan en el suelo y afectaran el crecimiento y desarrollo de las plantas de tomate, como lo refiere Villafañe [37] en plantas de batata (Ipomoea batata L.), en las que se acumularon $\mathrm{Na}$ y $\mathrm{Cl}$ por falta de mecanismos para exclusión de estos elementos, ocasionando restricción de agua y deterioro de la parte aérea y raíces, así como deformación de hojas.

Antes del trasplante el Fe tuvo incremento en las parcelas tratadas con $\mathrm{T}_{8}$ con respecto al valor alcanzado donde se aplicaría solución Steiner (Tabla 4), el cual llegó a ser de 7.5\% sin diferencia estadística entre ambos valores, mientras que con relación al valor estimado donde se aplicó $\mathrm{T}_{4}$ el incremento fue de $17.1 \%$ con diferencia estadística. Después de la cosecha los valores más altos se lograron donde se aplicó solución Steiner, $\mathrm{T}_{7}$ y $\mathrm{T}_{8}$, los cuales superaron en los respectivos 23.1\%, $18.7 \%$ y $21.7 \%$ al valor alcanzado con $\mathrm{T}_{5}$; entonces de acuerdo a la información de Salisbury y Ross [36], el hierro siempre estuvo presente en el suelo, de donde fue absorbido para formar parte del sitio catalítico de muchas enzimas óxido-reductoras, y de esa manera influir de manera importante en la formación de clorofila. Además, como lo menciona Hinrich [8], seguramente estuvo involucrado en la capacidad redox del suelo.

El contenido de Ti antes del trasplante fue más alto (21.3\%) donde se aplicó $\mathrm{T}_{8}$ comparado con el valor estimado donde se aplicó $\mathrm{T}_{4}$ (Tabla 4), y después de la cosecha continuó siendo el más alto sin diferencias estadísticas con respecto a los demás valores.

En cambio, el mayor porcentaje de $\mathrm{Al}$ antes del trasplante y después de la cosecha se obtuvo donde se aplicaría o se aplicó $\mathrm{T}_{1}$, sólo con incrementos de $16.6 \%, 12.6 \%, 14.4 \%, 14.2 \%, 19.7 \%$ y 19.9\% en comparación a los respectivos valores detectados donde se aplicaron $\mathrm{T}_{2}, \mathrm{~T}_{4}, \mathrm{~T}_{5}, \mathrm{~T}_{6}, \mathrm{~T}_{7} \mathrm{y} \mathrm{T}_{9}$ antes del trasplante, en tanto que después de la cosecha los incrementos fueron de $18.4 \%, 16.6 \%, 15.9 \%, 25.7 \%, 17.0 \%$ y $18.6 \%$ en comparación a lo detectado donde se adicionaron $\mathrm{T}_{2}, \mathrm{~T}_{3}, \mathrm{~T}_{4}, \mathrm{~T}_{5}, \mathrm{~T}_{6}$ y $\mathrm{T}_{9}$, respectivamente. El valor del Si no varió estadísticamente antes del trasplante, pero tuvo incrementos de 3.0\% - 9.5\% sobre los valores obtenidos con los demás de tratamientos; sin embargo, después de la cosecha lo detectado tuvo las diferencias estadísticas que se observan en la Tabla 4 con incrementos de $13.5 \%, 12.7 \%, 12.6 \%$, 20.2\%, 10.4\% y $14.2 \%$, solamente en relación a los respectivos valores obtenidos con $T_{2}, T_{3}, T_{4}, T_{5}, T_{6}, y T_{9}$. Esto permite deducir que el Si es como lo refiere Epstein [38], un elemento benéfico o cuasi-esencial, o como lo señala Haynes [39], un elemento beneficioso para las plantas. Además, Miramontes et al. [40] encontraron que con $3.28 \mathrm{cmol} \mathrm{(+)} \mathrm{de} \mathrm{Si} \cdot \mathrm{kg}^{-1}$ de suelo se tuvo un efecto significativo y altamente significativo sobre la altura y las biomasas fresca y seca, respectivamente, de la planta de chícharo (Pisum sativum L.).

El-haber aplicado los tratamientos antes del trasplante ocasionó que el $\mathrm{pH}$ y la ORP se incrementaran en todos los casos (Tabla 5) con respecto a lo registrado después de la cosecha, de tal forma que con todos los tratamientos que incluyeron ya sea composta más los minerales primarios amorfos o composta más minerales primarios amorfos y microorganismos, el $\mathrm{pH}$ se incrementó significativamente en comparación a lo estimado en las parcelas tratadas con solución Steiner, y los incrementos oscilaron de 4.7\% - 7.3\%. Este incremento del pH coincide con lo reportado por Aguilera [41], quien ha mencionado que la MOS afecta al pH debido a los diversos grupos activos que aportan grados de acidez, a las bases de cambio y al contenido de nitrógeno presente en los residuos orgánicos aportados al suelo. Además, fueron muy similares a los reportados por Lopez et al. [42], ya que a una profundidad de 0 - $30 \mathrm{~cm}$ de suelo donde aplicaron 20, 30 y $40 \mathrm{t} \cdot \mathrm{ha}^{-1}$ de estiércol de bovino, caprino y composta en combinación con 4,8 y $12 \mathrm{t} \cdot \mathrm{ha}^{-1}$ de gallinaza, más la aplicación de 4 , 8 y $12 \mathrm{t} \cdot \mathrm{ha}^{-1}$ de gallinaza, obtuvieron $\mathrm{pH}$ con valores de 8.05 - 8.50 . 
Tabla 5. Variables químicas del suelo al terminar la cosecha. Ciclo agrícola 2011-2012, Culiacán, Sinaloa. México.

\begin{tabular}{ccccccccc}
\hline & \multicolumn{2}{c}{$\mathrm{pH}(\mathrm{H})$} & \multicolumn{2}{c}{$\mathrm{CE}\left(\mathrm{dS} / \mathrm{m}^{-1}\right)$} & \multicolumn{2}{c}{ CIC $\left(\mathrm{cmolc} \mathrm{kg}^{-1}\right)$} & \multicolumn{2}{c}{ ORP $(\mathrm{mV})$} \\
\cline { 2 - 8 } Trat. & Antes & Después & Antes & Después & Antes & Después & Antes & Después \\
\hline $\mathrm{T}_{1}$ & $7.766 \mathrm{a}$ & $7.795 \mathrm{~b}$ & $1.798 \mathrm{a}$ & $1.155 \mathrm{a}$ & $12.680 \mathrm{~b}$ & $4.296 \mathrm{c}$ & $147.680 \mathrm{abc}$ & $289.750 \mathrm{a}$ \\
$\mathrm{T}_{2}$ & $7.833 \mathrm{a}$ & $8.245 \mathrm{a}$ & $0.435 \mathrm{~d}$ & $0.502 \mathrm{~b}$ & $34.663 \mathrm{a}$ & $26.083 \mathrm{a}$ & $159.680 \mathrm{ab}$ & $219.000 \mathrm{~d}$ \\
$\mathrm{~T}_{3}$ & $7.933 \mathrm{a}$ & $8.365 \mathrm{a}$ & $0.403 \mathrm{~d}$ & $0.882 \mathrm{ab}$ & $26.893 \mathrm{a}$ & $20.063 \mathrm{ab}$ & $151.000 \mathrm{abc}$ & $216.000 \mathrm{~d}$ \\
$\mathrm{~T}_{4}$ & $7.800 \mathrm{a}$ & $8.160 \mathrm{a}$ & $1.349 \mathrm{ab}$ & $0.997 \mathrm{ab}$ & $27.543 \mathrm{a}$ & $26.105 \mathrm{a}$ & $173.000 \mathrm{a}$ & $233.750 \mathrm{~cd}$ \\
$\mathrm{~T}_{5}$ & $7.800 \mathrm{a}$ & $8.172 \mathrm{a}$ & $0.586 \mathrm{~cd}$ & $0.787 \mathrm{ab}$ & $35.358 \mathrm{a}$ & $27.390 \mathrm{a}$ & $161.680 \mathrm{ab}$ & $220.000 \mathrm{~d}$ \\
$\mathrm{~T}_{6}$ & $7.742 \mathrm{a}$ & $8.370 \mathrm{a}$ & $0.887 \mathrm{bcd}$ & $0.537 \mathrm{~b}$ & $30.598 \mathrm{a}$ & $23.035 \mathrm{a}$ & $126.000 \mathrm{bcd}$ & $239.000 \mathrm{bc}$ \\
$\mathrm{T}_{7}$ & $7.500 \mathrm{a}$ & $8.297 \mathrm{a}$ & $1.158 \mathrm{abc}$ & $0.557 \mathrm{ab}$ & $35.400 \mathrm{a}$ & $18.913 \mathrm{ab}$ & $173.680 \mathrm{a}$ & $229.250 \mathrm{~cd}$ \\
$\mathrm{~T}_{8}$ & $6.933 \mathrm{~b}$ & $8.212 \mathrm{a}$ & $1.284 \mathrm{ab}$ & $0.752 \mathrm{ab}$ & $12.623 \mathrm{~b}$ & $9.452 \mathrm{bc}$ & $116.000 \mathrm{~cd}$ & $256.750 \mathrm{~b}$ \\
$\mathrm{~T}_{9}$ & $7.466 \mathrm{a}$ & $8.252 \mathrm{a}$ & $1.617 \mathrm{a}$ & $0.640 \mathrm{ab}$ & $35.280 \mathrm{a}$ & $26.083 \mathrm{a}$ & $93.670 \mathrm{~d}$ & $240.750 \mathrm{cb}$ \\
$\mathrm{DMSH}$ & 0.482 & .268 & 0.781 & 0.614 & 12.361 & 12.275 & 40.010 & 18.318 \\
\hline
\end{tabular}

Medias con diferente letra en la columna, son estadísticamente diferentes (Tukey, 0.05).

Sin embargo, en cuanto al potencial óxido reducción (ORP) que también se incrementó con todos los tratamientos al término de la cosecha, en comparación a lo registrado antes del trasplante, el valor más alto se obtuvo donde se aplicó la solución Steiner, el cual superó de 12.8\% - 34.1\% a lo estimado con los demás tratamientos. Los valores más altos de ORP después de la cosecha significan que la oxidación se incrementó a los niveles indicados en la Tabla 5, debido a la materia orgánica contenida en el suelo más la que se adicionó con la composta, el contenido de oxígeno y la pérdida de electrones por algunos elementos. No obstante, los valores de $\mathrm{mV}$ encontrados en esta investigación estuvieron muy por debajo de 400, de tal manera que como lo señalan Iván et al. [9] la óxido-reducción no fue una limitante en la producción del tomate.

La CE disminuyó en el 66.7\% de los casos en comparación a lo estimado antes del trasplante, incluida la parcela donde se aplicó la solución Steiner (Tabla 5); mientras que después de la cosecha, los valores de la CIC disminuyeron en todos los casos con respecto a los valores obtenidos antes del trasplante, pero la mayor disminución (66.1\%) ocurrió donde se aplicó la solución Steiner. Sin embargo, los valores de CIC encontrados antes del trasplante fueron muy similares a los reportados por Ribón et al. [43], toda vez que éllos obtuvieron valores de 23.9 - $34.9 \mathrm{Cmol} \mathrm{(+)} \mathrm{kg}^{-1}$ en parcelas con 5, 10, 20, 30 años cultivados con caña de azúcar y fertilizadas con la fórmula 120-60-60. Además, se cumplió con lo recomendado por la NOM-021-RECNAT-2000 [44], ya que esta norma considera que la reserva nutrimental del suelo es abundante cuando la CIC es mayor de $25 \mathrm{Cmol}(+) \mathrm{kg}^{-1}$ de suelo.

En la Tabla 6 se puede observar que en las parcelas manejadas con solución Steiner o con $\mathrm{T}_{8}$, la producción de frutos extragrandes superó desde $127.0 \%$ hasta $428.2 \%$ más donde se aplicó solución Steiner, mientras que donde se aplicó $\mathrm{T}_{8}$ los incrementos oscilaron de 146.4\% - 399.1\%. En frutos grandes la mayor producción se logró con $\mathrm{T}_{8}$, pero el promedio alcanzado sólo superó en $142.3 \%, 139.9 \%$ y 146.7\% a los respectivos promedios obtenidos con $\mathrm{T}_{3}, \mathrm{~T}_{5}$ y $\mathrm{T}_{7}$. La producción de frutos medianos se expresó con mayor intensidad donde se aplicó $\mathrm{T}_{2}$, aunque estadísticamente el promedio sólo superó en $163.2 \%, 1.56 .6 \%$ y $140.4 \%$ a lo alcanzado en las parcelas tratadas con $\mathrm{T}_{1}, \mathrm{~T}_{5}$ y $\mathrm{T}_{9}$, respectivamente. El rendimiento de frutos chicos fue superior en las parcelas tratadas con $\mathrm{T}_{2} \mathrm{y} \mathrm{T}_{7}$, de tal manera que con $\mathrm{T}_{2}$ sólo se superó en $279.2 \%, 135.8 \%$ y $76.7 \%$ a los promedios obtenidos con $\mathrm{T}_{1}, \mathrm{~T}_{8}$ y $\mathrm{T}_{9}$, respectivamente; en tanto que con $\mathrm{T}_{7}$ los respectivos incrementos fueron de $277.3 \%, 134.9 \%$ y $138.7 \%$ con respecto a los mismos promedios. Sin embargo, la mayor producción total se obtuvo con $\mathrm{T}_{8}$, la cual no superó a lo logrado con la solución Steiner, pero en relación a los respectivos promedios cosechados con los tratamientos $\mathrm{T}_{2}, \mathrm{~T}_{3}, \mathrm{~T}_{4}, \mathrm{~T}_{5}, \mathrm{~T}_{6}, \mathrm{~T}_{7}$ y $\mathrm{T}_{9}$, se estimaron incrementos de $123.1 \%, 157.2 \%, 142.7 \%, 174.0 \%, 140.9 \%$, $154.5 \%$ y $126.7 \%$.

La producción total de $114 \mathrm{Mg} \cdot \mathrm{ha}^{-1}$ obtenida de las parcelas tratadas con $25 \mathrm{Mg} \cdot \mathrm{ha}^{-1}$ de composta $+6 \mathrm{Mg} \cdot \mathrm{ha}^{-1}$ de MPA $\left(\mathrm{T}_{8}\right)$ casi fue similar a lo que Parra et al. [45] obtuvieron en otra investigación (113.97 Mg $\cdot \mathrm{ha}^{-1}$ ) con el mismo cultivar, pero superó a lo reportado por Preciado et al. [46], consistente en 128, 60.9, 101.6 y 54.6 
Tabla 6. Producción y calidad de tomate saladette: extra grande, grande, mediano, chico y producción total, con el uso de solución Steiner, composta, minerales primarios amorfos y consorcio microbiano.

\begin{tabular}{cccccc}
\hline Tratamientos & Extragrande $\left(\mathrm{kg} \cdot \mathrm{m}^{-2}\right)$ & Grande $\left(\mathrm{kg} \cdot \mathrm{m}^{-2}\right)$ & Mediano $\left(\mathrm{kg} \cdot \mathrm{m}^{-2}\right)$ & Chico $\left(\mathrm{kg} \cdot \mathrm{m}^{-2}\right)$ & Total $\left(\mathrm{kg} \cdot \mathrm{m}^{-2}\right)$ \\
\hline $\mathrm{T}_{1}$ & $5.010 \mathrm{a}$ & $3.220 \mathrm{ab}$ & $1.660 \mathrm{~b}$ & $0.530 \mathrm{~d}$ & $10.430 \mathrm{ab}$ \\
$\mathrm{T}_{2}$ & $1.830 \mathrm{c}$ & $3.250 \mathrm{ab}$ & $2.710 \mathrm{a}$ & $1.480 \mathrm{a}$ & $9.260 \mathrm{bc}$ \\
$\mathrm{T}_{3}$ & $1.560 \mathrm{c}$ & $2.340 \mathrm{c}$ & $2.050 \mathrm{ab}$ & $1.330 \mathrm{abc}$ & $7.250 \mathrm{~cd}$ \\
$\mathrm{~T}_{4}$ & $1.850 \mathrm{c}$ & $2.630 \mathrm{abc}$ & $2.160 \mathrm{ab}$ & $1.360 \mathrm{ab}$ & $7.990 \mathrm{~cd}$ \\
$\mathrm{~T}_{5}$ & $1.170 \mathrm{c}$ & $2.380 \mathrm{bc}$ & $1.730 \mathrm{~b}$ & $1.280 \mathrm{abc}$ & $6.550 \mathrm{~d}$ \\
$\mathrm{~T}_{6}$ & $1.970 \mathrm{c}$ & $2.710 \mathrm{abc}$ & $2.120 \mathrm{ab}$ & $1.280 \mathrm{abc}$ & $8.090 \mathrm{~cd}$ \\
$\mathrm{~T}_{7}$ & $1.330 \mathrm{c}$ & $2.270 \mathrm{c}$ & $2.310 \mathrm{ab}$ & $1.470 \mathrm{a}$ & $7.380 \mathrm{~cd}$ \\
$\mathrm{~T}_{8}$ & $4.670 \mathrm{a}$ & $3.330 \mathrm{a}$ & $2.310 \mathrm{ab}$ & $1.090 \mathrm{bc}$ & $11.400 \mathrm{a}$ \\
$\mathrm{T}_{9}$ & $3.190 \mathrm{~b}$ & $2.820 \mathrm{abc}$ & $1.930 \mathrm{~b}$ & $1.060 \mathrm{c}$ & $9.000 \mathrm{bc}$ \\
\hline
\end{tabular}

Medias con diferente letra en la columna, son estadísticamente diferentes (Tukey, 0.05).

$\mathrm{Mg} \cdot \mathrm{ha}^{-1}$, que obtuvieron de tomate saladette cultivado con solución Steiner, té de composta, té de vermicomposta y lixiviado de vermicomposta, respectivamente. También superó la producción de $84 \mathrm{Mg} \cdot \mathrm{ha}^{-1}$ que obtuvieron Cun et al. [47], mediante aplicación de hongos micorrizógenos del género Glomus y humus de lombriz. Lo mismo ocurrió con el rendimiento obtenido por De la Cruz et al. [48], quienes sólo cosecharon $39.8 \mathrm{Mg} \cdot \mathrm{ha}^{-1}$ de donde aplicaron composta de estiércol de bovino, rastrojo de maíz, zacate elefante y tierra negra (CEMZT) al 75\% + arena y la vermicomposta de estiércol, pasto bahía y tierra negra (VEPT) al 100 y $50 \%$ + arena. Las $57.4 \mathrm{Mg} \cdot \mathrm{ha}^{-1}$ obtenidas por De la Cruz et al. [49] a partir del tomate cultivado con la relación (1:1:1, V:V:V) de la mezcla vermicomposta de estiércol de ganado vacuno + paja de maíz y tierra negra $(75 \%+25 \%$ de arena), también fueron superadas por la producción que se logró en esta investigación.

No obstante, la producción de $250 \mathrm{Mg} \cdot \mathrm{ha}^{-1}$ que obtuvieron Ortega et al. [50] de los tomates (seis plantas $\cdot \mathrm{m}^{-2}$ ) cultivados en sustrato formado de aserrín de pino composteado + composta de estiércol de ovino y solución Steiner, fue superior a la que se obtuvo en esta investigación. Asimismo, con respecto a la producción reportada por Cruz et al. [51], la cual fue de $146.33 \mathrm{Mg} \cdot \mathrm{ha}^{-1}$ a partir del híbrido "Charleston" cultivado en mezcla de tezontle + vermicomposta de estiércol bovino y desechos vegetales. También fue inferior a las $136.7 \mathrm{Mg} \cdot \mathrm{ha}^{-1}$ que cosecharon Márquez et al. [52], cuando fertilizaron las variedades de tomate Big Beef y Bosky con solución inorgánica y composta + macro y micro elementos inorgánicos. La influencia de los microorganismos no se notó porque quizás las plantas de tomate no necesitaron nutrientes más allá de los que se mineralizaron a partir del $0.86 \%$ de MO ya existente en el suelo, de la composta y los minerales primarios amorfos adicionados.

\section{Conclusiones}

La fertilización con $25 \mathrm{Mg} \cdot \mathrm{ha}^{-1}$ de composta y $6 \mathrm{Mg} \cdot \mathrm{ha}^{-1}$ de MPA fue suficiente para que con el híbrido "Moctezuma" de tomate saladette se obtuviera una producción de similar proporción a la que se puede lograr con solución nutritiva Steiner.

La incorporación de composta, MPA y microorganismos hizo que el suelo incrementara su fertilidad desde antes del trasplante, y hasta la cosecha se continuara proveyendo de suficientes nutrientes para el crecimiento, desarrollo y rendimiento de tomate, con excepción del $\mathrm{Cl}$ y el S, el primero que apenas se detectó antes del trasplante, y el segundo que ya no fue detectado después de la cosecha, al igual que $\mathrm{Cl}$.

Las deficiencias de $\mathrm{Cl}$ y $\mathrm{S}$ en el suelo antes del trasplante y después de la cosecha quizás fueron unas de las causas por las que las plantas de tomate ya no produjeron más allá de lo que se logró con la solución Steiner y con $\mathrm{T}_{8}$, ya que el S es un elemento importante en la formación de compuestos orgánicos (proteínas, cistina, cisteína, metionina, etc.), mientras que el $\mathrm{Cl}$ forma parte del Fotosistema II que es importante para la fotosíntesis, además de formar parte de más de 130 compuestos orgánicos, dentro de éllos el ácido 4-cloroindolacético, que es una hormona del tipo de auxina.

Los porcentajes de nutrimentos detectados antes de que aplicara solución Steiner en las parcelas, indicaron que 
en el suelo ocurrieron la oxidación y reducción química de nutrimentos de manera natural, y que después que se aplicaron todos los tratamientos los dos fenómenos siguieron dándose, aunque la reducción fue más intensa en algunos elementos que otros, para que muchos de los nutrientes estuvieran disponibles para las plantas de tomate, excepto el $\mathrm{Cl}$, antes del trasplante y después de la cosecha.

La no detección de $\mathrm{S}$ después de la cosecha en todas las parcelas, y la del Zn en algunas parcelas, indicó que estos son elementos en los que la reducción fue muy prominente, pero que fue menor en relación a la demanda de las plantas, ocasionando que aparentemente se agotaran al final de la cosecha de tomate.

A través de la fertilización con $\mathrm{T}_{8}\left(25 \mathrm{Mg} \cdot \mathrm{ha}^{-1}\right.$ de composta y $6 \mathrm{Mg} \cdot \mathrm{ha}^{-1}$ de MPA) se pudo llevar a cabo el manejo del suelo y plantas de manera más sustentable que cuando sólo se manejaron con solución Steiner, puesto que con $\mathrm{T}_{8}$ se aportó materia orgánica y minerales, de donde continuaron las reducciones químicas para seguir proporcionando nutrimentos asimilables por parte de las plantas.

\section{References}

[1] FitzPatrick, E.A. (1996) Introducción a la Ciencia de los Suelos. Primera edición en español. Editorial S. A de C.V., $161 \mathrm{p}$.

[2] Stamford, N.P., Moura, P.M., Lira, J.M.A., Santos, C.E.deR.S., Duenhas, L.H. and Gava, C.A. (2009) Chemical Attributes of an Argisoil of the Vale do São Francisco after Melon Growth with Phosphate and Potash Rocks Biofertilizers. Horticultura Brasileira, 27, 447-452. http://dx.doi.org/10.1590/S0102-05362009000400008

[3] FAO (2015) Los suelos sanos son la base para la producción de alimentos saludables. http://www.fao.org/soils-2015/news/news-detail/es/c/277721/

[4] Curtis, H.and Sue, B.N. (2001) Biología. Médica Panamericana, Buenos Aires, Argentina, 1449-1450.

[5] Fournier, O. and Luis, S. (2003) Recursos Naturales. Sexta reimpresión. Editorial Universidad a Distancia, San José, Costa Rica, 216 p.

[6] Bertrand, J.C., Caumette, P., Lebaron, P., Matheron, R., Normand, P. and Sime-Ngando, T. (2015) Environmental Microbiology: Fundamentals and Applications: Microbial Ecology. Springer Science + Business Media, Dordrecht, 511 p.

[7] Urtis, H. and Barnes, N.S. (2001) Biología. Capítulo 7. El flujo de energía. Sexta Edición en español. Editorial Médica Panamericana S.A., 189.

[8] Hinrich, L.B., Brian, L.M. and George, A.O. (2002) Soil Chemistry. 3rd Edition. Editorial John Wiley \& Sons, Ontario, $320 \mathrm{p}$.

[9] Iván, G.M., Castro, H.E. and Malagón, D. (2005) Interpretación de procesos redox en suelos sulfatados ácidos del distrito de riego del Alto Chicamocha, Boyacá. Agronomía Colombiana, 23, 136-142.

[10] Miller, H.G. and Augustine, B.F. (1978) Química Básica. Harper y Row Latinoamericana, EditoriaL·harla S. A. de C. V. México 4. Distrito Federal, 256-257.

[11] Páez, O.F. (2007) Contaminación por nitrógeno y fósforo en Sinaloa: Flujos, fuentes, efectos y opciones de manejo. Serie Lagunas Costeras de Sinaloa 2. Instituto de Ciencias del Mar y Limnología, Universidad Nacional Autónoma de Sinaloa, 140.

[12] Rosset, P., Patel, R. and Courville, M. (2006) Promised Land: Competing Visions of Agrarian Reform. USDA, Food First Books, Oakland, 380 p.

[13] Espinoza, O.R. (1996) La agricultura Moderna: Sus Retos, Cambios y Perspectivas. Editorial Universidad Autónoma de Sinaloa, Sinaloa, 179 p.

[14] Martínez, H.E., Fuentes, E.J.P. and Acevedo, H.E. (2008) Carbono orgánico y propiedades del suelo. Revista de la Ciencia del Suelo y Nutrición Vegetal, 8, 68-96.

[15] Novella, L.R. (2001) Participación de las micorrizas arbusculares y la fertilización nitrogenada en el crecimiento y producción de tomate (Lycopersicon esculentum Mill.) en un suelo ferralsol desaturado. Tesis de Maestría en Ciencias en Nutrición de Plantas y Biofertilizantes, Universidad de Granma, Bayamo, 63 p.

[16] Pocknee, S. and Sumner, M.E. (1997) Carbon and Nitrogen Contents of Organic Matter Determine Its Soil Liming Potential. Soil Scince Society of America Journal, 61, 86-92. http://dx.doi.org/10.2136/sssaj1997.03615995006100010014x

[17] García, A.E. (1988) Modificaciones al sistema de clasificación climática de Köppen. Instituto de Geografía. UNAM, Mexico City, 217 p.

[18] Steiner, A.A. (1961) A Universal Method for Preparing Nutrient Solutions of a Certain Desired Composition. Plant and Soil, 15, 134-154. http://dx.doi.org/10.1007/BF01347224

[19] Eghball, B., Wienhold, B.J., Gilley, J.E. and Eigenberg, R.A. (2002) Mineralization of Manure Nutrients. Journal of 
Soil and Water Conservation, 57, 469-473.

[20] Quero, G.E. (2010) La biosilicificación proceso biológico fundamental en la productividad vegetal. Revista de Riego: Protección y Nutrición de Hortalizas y Frutas. Año 6, No. 39, 72-76.

[21] FAO (2002) Captura de carbono en los suelos para un mejor manejo de la tierra. http://www.fao.org/docrep/005/y2779s/y2779s05

[22] Craswell, E.T. and Lefroy, R.B.D. (2000) The Role and Function of Organic Matter in Tropical Soils. Nutrient Cycling in Agroecosystems, 61, 7-18. http://dx.doi.org/10.1023/A:1013656024633

[23] Singer, M.J. and Munns, D.N. (1996) Soils: An Introduction. Third Edition, Prentice Hall, Publishing Co., Upper Saddle River, USDA, 480 p.

[24] Oades, J.M., Gillman, G.P. and Uehara, G. (1989) Interactions of Soil Organic Matter and Variable-Charge Clays. In: Coleman, D.C., Oades, J.M. and Uehara, G., Eds., Dynamics of Soil Organic Matter in Tropical Ecosystems, University of Hawaii Press, Honolulu, 69-95.

[25] Krull, E.S., Skjemstad, J.O. and Baldock, J.A. (2004) Functions of Soil Organic Matter and the Effect on Soil Properties. Grains Research \& Development Corporation Report Proyect No. CSO 00029.

[26] Cajuste, L.J. (2007) Química de suelos con enfoque agrícola. Colegio de Postgraduados, Chapingo, México, 278.

[27] Fassbender, H. and Bornemisza, E. (1994) Química de suelos con énfasis en suelos de América Latina. Segunda edición revisada. San José Costa Rica, 420.

[28] Takkar, P.N. (1988) Sulfur Status of Indian Soils. Proc. The Sulphur Institute Fertilizer Association of India Symp. Sulfur in Indian Agriculture, New Delhi, 5/1/2/1-31.

[29] Tisdale, S.L. and Nelson, W.L. (1991) Fertilidad de los suelos y fertilizantes. Editorial Limusa, 760 p.

[30] Prasad, R. and Power, J.F. (1997) Soil Fertility Management for Sustainable Agriculture. Lewis Publishers in an Imprint of CRC Press, 243.

[31] Nuez, F. (2001) El Cultivo de Tomate. Editorial Mundi-Prensa, Madrid, 793 p.

[32] Messick, D.L., Fan, M.X. and Brey, C. (2005) Global Sulfur Requirement and Sulfur Fertilizer. Número Especial. 283. In: De Kok, L. and Schnug, E., Eds., Proceedings of the first Sino-German Workshop on Aspect of Sulfur Nutrition of Plants, 23-27 Mayo 2004, Shenyang, 97-104.

[33] Navarro, G. (2003) Química agrícola. El suelo y los elementos químicos esenciales para la vida vegetal. Segunda Edición, Ediciones Mundi-Prensa, New York, London, Oxford, 15-38.

[34] Vallee, B.L. (1976) Zinc Biochemistry: A Perspectiva. Trends in Biochemical Sciences, 1, 88-91.

[35] Navarro, G.G. and Navarro, G.S. (2013) Química Agrícola. Química del suelo y de los nutrientes esenciales para las plantas. 3ra Edición. Ediciones Mundi-Prensa, Impreso en España, 453.

[36] Salisbury, F.B. and Ross, C.W. (2000) Fisiología de las Plantas. Paraninfo Thomson Learning, Madrid, España, 988 p.

[37] Villafañe, R. (1997) Efecto de la salinidad del suelo sobre el crecimiento de la batata. Agronomía Tropical, 47, 131139.

[38] Epstein, E. (1999) Silicon. Annual Review of Plant Physiology and Plant Molecular Biology, 50, 641-664. http://dx.doi.org/10.1146/annurev.arplant.50.1.641

[39] Haynes, R.J. (2014) A Contemporary Overview of Silicon Availability in Agricultural Soils. Journal of Plant Nutrition and Soil Science, 177, 831-844. http://dx.doi.org/10.1002/jpln.201400202

[40] Miramontes, F.B., Arroyo, V.L., Alva, R.M.H. and Espericueta, R.T. (2004) Efecto del metasilicato de sodio sobre el crecimiento del cultivo de chícharo. Terra Latinoamericana, 22, 169-174.

[41] Aguilera, S.M. (2000) Importancia de la protección de la materia orgánica en suelos. Simposio Proyecto Ley Protección de Suelo. Boletín No. 14. Valdivia, 77-85.

[42] López, M.J., Díaz, E.A., Martínez, R.E. and Valdez, C.R. (2001) Abonos orgánicos y su efecto en propiedades físicas y químicas del suelo y rendimiento en maíz. TERRA Latinoamericana, 19, 293-299.

[43] Ribón, C.M.A., Salgado, G.S., Palma, L.D.J. and Lagunes, E.L.C. (2003) Propiedades químicas y físicas de un vertisol cultivado con caña de azúcar. Interciencia, 28, 154-159.

[44] NOM-021-RECNAT-2000 (2002) Secretaria de Medio Ambiente y Recursos Naturales. Segunda Sección, 85.

[45] Parra, D.J.M., Velázquez, A.T.J., Quero, G.E., Partida, R.L, Díaz, V.T, Galván, P.B. and Ayala, T.F. (2014) Uso de composta, minerales primarios amorfos y microorganismos para la producción y calidad de tomate. Revista Intrópica, 9, 102-110.

[46] Preciado, R.P., Fortis, M., García, H.J.L., Rueda, E.O., Esparza, J.R., Lara, A., Segura, M.A. and Orozco, J.A. (2011) Evaluación de soluciones nutritivas orgánicas en la producción de tomate en invernadero. Interciencia, 36, 689-693. 
[47] Cun, G.R., Duarte, D.C. and Montero, S.L. (2008) Producción orgánica de tomate mediante la aplicación de humus de lombriz y EcoMic ${ }^{\circledR}$ en condiciones de casa de cultivo. Revista Ciencias Técnicas Agropecuarias, 17, 22-25.

[48] De la Cruz, L.E., Botello, M.A.E., Robledo, T.V., Osorio, O.R., Márquez, H.C. and Sánchez, H.R. (2009) Producción de tomate en invernadero con composta y vermicomposta como sustrato. Universidad y Ciencia, 25, 59-67.

[49] De la Cruz, L.E., Osorio, O.R., Martínez, M.E., Lozano del Rio, A.J., Gómez, V.A. and Sánchez, H.R. (2010) Uso de compostas y vermicompostas para la producción de tomate orgánico en invernadero. Interciencia, 35, 363-368.

[50] Ortega, M.L.D., Sánchez, D.O.J., Ocampo, M.J., Sandoval, C.E., Salcido, R.B.A. and Manzo, R.F. (2010) Efecto de diferentes sustratos en crecimiento y rendimiento de tomate (Lycopersicum esculentum Mill.) bajo condiciones de invernadero. Ra Ximhai, 6, 339-346.

[51] Cruz, C.E., Sandoval, V.M., Volke, H.V.H., Can, C.A. and Sánchez, E.J. (2012) Efecto de mezclas de sustratos y concentración de la solución nutritiva en el crecimiento y rendimiento de tomate. Revista Mexicana de Ciencias Agrícolas, 3, 1361-1373.

[52] Márquez-Hernández, C., Cano-Ríos, P., Figueroa-Viramontes, U., Avila-Diaz, J.A., Rodríguez-Dimas, N., GarcíaHernández, J.L. (2013) Yield and Quality of Tomato with Organic Sources of Fertilization under Greenhouse Conditions. International Journal of Experimental Botany, 82, 55-61. 